\title{
Organotellurium Chemistry: Synthesis and Properties of 2-Acylamino- and 2-Arylamino-1,3-benzotellurazoles
}

\author{
Whitney Elisabeth Smith ${ }^{1}$, Donna Victoria Franklin ${ }^{1}$, Kourtni Lynn Goutierrez ${ }^{1}$, \\ Frank Robert Fronczek ${ }^{2}$, Franz Andreas Mautner ${ }^{3}$, Thomas Junk ${ }^{1, *}$ \\ ${ }^{1}$ Department of Chemistry, University of Louisiana at Lafayette, Lafayette, USA \\ ${ }^{2}$ Department of Chemistry, Louisiana State University, Baton Rouge, USA \\ ${ }^{3}$ Institute for Physical and Theoretical Chemistry, Technical University Graz, Graz, Austria
}

Email address:

txj9137@louisiana.edu (T. Junk)

*Corresponding author

\section{To cite this article:}

Whitney Elisabeth Smith, Donna Victoria Franklin, Kourtni Lynn Goutierrez, Frank Robert Fronczek, Franz Andreas Mautner, Thomas Junk. Organotellurium Chemistry: Synthesis and Properties of 2-Acylamino- and 2-Arylamino-1,3-benzotellurazoles. American Journal of Heterocyclic Chemistry. Vol. 5, No. 3, 2019, pp. 49-54. doi: 10.11648/j.ajhc.20190503.11

Received: December 6, 2019; Accepted: July 10, 2019; Published: July 30, 2019

\begin{abstract}
Synthetic methods have been developed to prepare novel 1,3-benzotellurazoles carrying acylamino and arylamino moieties in position 2, in order to investigate their propensity to self-assemble to supramolecular structures. The targeted compounds were obtained in yields ranging from $44 \%$ to $67 \%$, by reacting bis(2-aminophenyl) ditelluride with acyl- and aryl isothiocyanates, respectively, and subsequent reductive cyclization of the resulting thiourea derivatives. Seven novel 1,3benzotellurazole derivatives were prepared: 2-benzoylamino-1,3-benzotellurazole, 2-(4-chlorobenzoylamino)-1,3benzotellurazole, 2-(2-bromobenzoylamino)-1,3-benzotellurazole, 2-(4-bromobenzoylamino)-1,3-benzotellurazole, 2-(4methoxybenzoylamino)-1,3-benzotellurazole, 2-phenylamino-1,3-benzotellurazole, and 2-(4-chlorophenylamino-1,3benzotellurazole. A simplified protocol was employed to synthesize all acyl isothiocyanates needed for their preparation from benzoyl halide derivatives and potassium thiocyanate. The reductive cyclization of the intermediate thioureas was challenging, only the use of hydroxymethanesulfinate in the presence of elemental mercury provided synthetically useful product yields. A mechanism was proposed, consisting of the insertion of mercury into the Te-Te bond, followed by intramolecular nucleophilic attack of the thiocarbonyl moiety by the resulting insertion product. All 2-acylamino-1,3-benzotellurazoles are crystalline solids, which are stable to ambient light, air and moderate heat. A characterization of selected samples by X-ray crystallography indicated that they form dimers in solid state, resulting from hydrogen bonding between the exocyclic and endocyclic nitrogen atoms of two adjacent molecules. This sets them apart from 2-alkyl- and 2-aryl-1,3-benzotellurazoles, which are known to self-assemble into supramolecular wires.
\end{abstract}

Keywords: Tellurium, Tellurazoles, Self-Assembly

\section{Introduction}

The chemistry of the five-membered Te, N-containing heterocycles has found recent interest, largely due to their tendency to self-assemble into supramolecular structures. In this respect, the 1,2,5-telluradiazoles and isotellurazole $\mathrm{N}$ oxides have been at the focus of prior work; however, the sensitivity to hydrolysis of the former and low yield access to the latter make the availability of alternatives desirable [1-3]. 1,3-Benzotellurazoles are attractive in this respect, due to their high stability towards air, light and heat, as well as their established ability to form supramolecular wires via intermolecular Te-N coordination [4-6] (Figure 1).

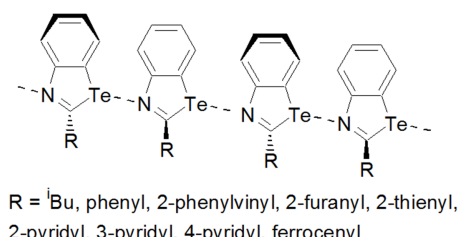

Figure 1. Molecular wires formed from 1,3-benzotellurazoles. 
Recent advances in the preparation of 1,3benzotellurazoles [7] have drastically improved access to this class of compounds. Their ability to form aggregates is influenced by the presence and nature of substituents. This prompted us to seek improved synthetic access to 2-amino and 2-acylamino substituted 1,3-benzotellurazoles, to investigate the effects, which the exocyclic nitrogen has on self-assembly. Access to these compounds via the reaction of 2-iodophenylisocyanide with elemental tellurium and the lithium salts of secondary amides at low temperature has been reported, but is somewhat challenging and narrow in scope [8]. Consequently, we investigated ring closures analogous to those previously reported for phenols and thiophenols carrying thiourea moieties in ortho position, which were converted to benzoxazoles and benzothiazoles by heating in the presence nickel peroxide [9], lead oxide [10] or mercuric oxide [11] in moderate to good yields (Figure 2).

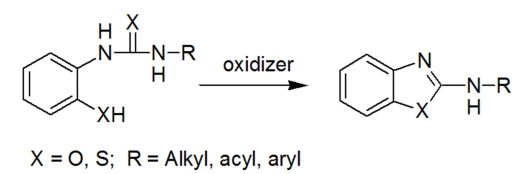

Figure 2. Access to benzoxazoles and benzothiazoles.

Remarkably, no reference for the analogous preparation of 2-amino substituted selenazoles could be found. Indeed, the increasing tendency of the heavier chalcogen congeners of phenol to oxidize makes it essentially impossible to carry out reactions under oxidative conditions without their simultaneous oxidation to the corresponding dichalcogenides. Furthermore, the difficulty of preparing and handling arenetelluroles makes their direct use impractical [12]. Instead, they usually are generated in situ. Consequently, bis(2-aminophenyl) ditelluride was selected as precursor compound for the targeted products. Its reaction with aryl- or acylisothiocyanates furnished thioureas, which underwent reductive desulfurization when treated with sodium hydroxymethanesulfinate (Rongalite) in the presence of elemental mercury in refluxing dimethylformamide (DMF).

\section{Materials and Methods}

Bis(2-aminophenyl) ditelluride was prepared as previously reported [7]. 4-Chlorophenyl isothiocyanate was prepared following a published procedure [13]. Benzoyl isothiocyanate, 2-bromobenzoyl isothiocyanate, 4methoxybenzoyl isothiocyanate and 4-chlorobenzoyl isothiocyanate were prepared in analogy to a procedure published for benzoyl isothiocyanate [14], with modifications as stated below. All other chemicals were purchased as reagent grade and used as received. Silica gel 40-65 $\mu \mathrm{m}$, obtained from VWR, was used for flash chromatography. Melting points were recorded in open capillaries using an electrothermal SRS MPA160 apparatus and are not corrected. Nuclear magnetic resonance spectra were recorded on a Varian MR $400 \mathrm{MHz}$ NMR spectrometer. High resolution mass spectra were recorded on an Agilent 6230 ESI TOF mass spectrometer. Peaks in the $[\mathrm{M}+\mathrm{H}]^{+}$cluster exceeding $20 \%$ of base are listed. Infrared spectra were recorded for pure solids with a Jasco FTIR-4700 spectrometer. Caution: Due the use of elemental mercury and the possible generation of trace amounts of hydrogen sulfide and volatile organotellurium compounds, all operations should be carried out under a well vented hood. Proper disposal procedures are required for mercury-containing waste products.

\subsection{Experimental Section}

\subsubsection{Benzoyl Isothiocyanate (1a), a Representative Procedure for the Synthesis of Aryl Isothiocyanates (1a-e)}

A $25 \mathrm{~mL}$ round bottom flask equipped with a magnetic stirring bar was charged with mixture of benzoyl chloride (4.22 g, $30 \mathrm{mmol})$, oven dried potassium thiocyanate $(4.37 \mathrm{~g}$, $45 \mathrm{mmol}$ ) and $8 \mathrm{~mL}$ of benzene. The flask was capped and stirred for two days at room temperature under exclusion of moisture. The solvent was subsequently removed by rotary evaporation and the residue extracted with $210 \mathrm{~mL}$ of dichloromethane. Solids were removed by centrifugation due to the presence of very fine particulate matter. The solvent was removed by rotary evaporation at $40^{\circ} \mathrm{C}$ and the residue extracted with $2 \times 10 \mathrm{~mL}$ of warm hexane. A fine turbidity was removed from the extract by centrifugation, followed by rotary evaporation at $40^{\circ} \mathrm{C}$. Benzoyl isothiocyanate (1a) was obtained as pale yellow oil. It was characterized by GC-MS analysis and found to be $>90 \%$ pure. Its properties were identical to those reported by Kuzovlev [14]. It was stored at $4{ }^{\circ} \mathrm{C}$ and used without further purification. Yield, $3.28 \mathrm{~g}$ ( $67 \%$ of theory).

Following the same procedure, the following isothiocyanates were prepared: 2-bromobenzoyl isothiocyanate (1b), 75\% yield; 4-methoxybenzoyl isothiocyanate (1c), 61\% yield; 4-chlorobenzoyl isothiocyanate (1d), 64\% yield, and 4bromobenzoyl isothiocyanate (1e), 78\% yield. All compounds were confirmed by GC-MS analysis to be $>90 \%$ pure, with properties matching those previously published [15-17]. No attempts of further purification were made, due to their tendency to slowly decompose. All products were stored at $4^{\circ} \mathrm{C}$ and used as soon as feasible.

\subsubsection{2-Benzoylamino-1,3-benzotellurazole (2a), a Representative Procedure for the Synthesis of 1,3-benzotellurazoles (2a-f)}

A $25 \mathrm{~mL}$ round bottom flask with magnetic stirring was charged with bis(2-aminophenyl) ditelluride (132 mg, 0.30 $\mathrm{mmol}$ ) and benzoylisothiocyanate (107.7 $\mathrm{mg}, 0.66 \mathrm{mmol})$, dissolved in $1.2 \mathrm{~g}$ of dimethylformamide (DMF). The mixture was stirred until homogeneous and subsequently kept at $80^{\circ} \mathrm{C}$ in a drying oven for 2 hours. A color change from red to dark orange was observed as the thiourea formed. The flask was subsequently fitted with a heating mantle and a reflux condenser, elemental mercury $(180 \mathrm{mg}, 0.9 \mathrm{mmol})$ and Rongalite $(78 \mathrm{mg}, 0.66 \mathrm{mmol})$ were added and the mixture heated to reflux with rapid stirring. Heating was continued for 90 minutes. The content of the flask was subsequently allowed to cool to room temperature, transferred to a centrifuge tube 
and diluted with $20 \mathrm{~mL}$ of water. The crude product and insoluble byproducts separated as extremely fine black-gray solid, which required 30 minutes of centrifugation at 4,400 rpm to achieve satisfactory phase separation. The supernatant aqueous phase was discarded and the residue washed with another $20 \mathrm{~mL}$ of water, followed by centrifugation as before to remove any residual DMF. The product was subsequently extracted with $2 \times 8 \mathrm{~mL}$ of dichloromethane (DCM), again using centrifugation to achieve satisfactory phase separation. The combined organic phases were further purified by flash chromatography $(8 \mathrm{~mm} \times 2 \mathrm{~cm}$ silica gel column, mobile phase DCM) and volatiles allowed evaporate under a hood in an open beaker to permit evaporation/oxidation of traces of malodorous byproducts. The products were crystallized from toluene, then from ethanol.

\subsection{Characterization of Synthesized Compounds (2a-j)}

\subsubsection{2-Benzoylamino-1,3-benzotellurazole (2a)}

Colorless crystals, M.F.: $\mathrm{C}_{14} \mathrm{H}_{10} \mathrm{~N}_{2} \mathrm{OTe}$; Yield: $56 \%$; M.W.: 349.84; M.P. (K): 496-498; FTIR (cm $\left.{ }^{-1}\right): 2704$ (C-H), 1595, 1518, 1329, 1236 (aromatic); ${ }^{1} \mathrm{H}$ NMR $\left(\mathrm{CDCl}_{3}\right): \delta 7.17(\mathrm{t}$, $1 \mathrm{H}), 7.34-7.57(\mathrm{~m}, 5 \mathrm{H}) ; 7.90(\mathrm{~d}, 1 \mathrm{H}), 8.07(\mathrm{~d}, 2 \mathrm{H}), 8.80(\mathrm{~N}-$ $\mathrm{H}) ;{ }^{13} \mathrm{C} \mathrm{NMR}\left(\mathrm{CDCl}_{3}\right): \delta 120.35,124.25,126.45,127.26$, $128.26,128.83,131.17,132.81,133.20,146.84,163.77$, 170.03; MS (m/z, \%): $[\mathrm{M}+\mathrm{H}]^{+} 347.9912$ (22.72), 348.9902 (58.04), 350.9911 (92.71), 352.9929 (100); Anal: C, 47.78; H, 2.70; N, 7.88.

\subsubsection{2-(4-Chlorobenzoylamino)-1,3-benzotellurazole (2b)}

Colorless crystals, M.F.: $\mathrm{C}_{14} \mathrm{H}_{9} \mathrm{ClN}_{2} \mathrm{OTe}$; Yield: 51\%; M.W.: 384.28; M.P. (K): 493-495; FTIR $\left(\mathrm{cm}^{-1}\right): 3133(\mathrm{C}-\mathrm{H})$, 1649, 1587, 1531 (aromatic); ${ }^{1} \mathrm{H}$ NMR $\left(\mathrm{CD}_{3} \mathrm{COCD}_{3}\right): \delta 7.16$ $(\mathrm{t}, 1 \mathrm{H}), 7.44(\mathrm{t}, 1 \mathrm{H}) ; 7.56(\mathrm{~d}, 2 \mathrm{H}), 7.76(\mathrm{~d}, 1 \mathrm{H}), 8.03(\mathrm{~d}, 1 \mathrm{H})$, $8.32(\mathrm{~d}, 2 \mathrm{H}), 12.31(\mathrm{~N}-\mathrm{H}) ;{ }^{13} \mathrm{C} \mathrm{NMR}\left(\mathrm{CD}_{3} \mathrm{COCD}_{3}\right): \delta 117.54$, $123.28,123.47,127.07,128.55,130.45,131.83,134.37$, $137.85,144.09,168.80,172.61 ; \mathrm{MS}(\mathrm{m} / \mathrm{z}, \%):[\mathrm{M}+\mathrm{H}]^{+}$ 382.9509 (48.28), $384.9515 \quad(85.83), 386.9528 \quad(100)$, 388.9513 (25.75); Anal: C, 44.03; H, 2.33; N, 7.39.

\subsubsection{2-(2-Bromobenzoylamino)-1,3-benzotellurazole (2c)}

Colorless crystals, M.F.: $\mathrm{C}_{14} \mathrm{H}_{9} \mathrm{BrN}_{2} \mathrm{OTe}$; Yield: $48 \%$; M.W.: 428.74; M.P. (K): 452-453; FTIR $\left(\mathrm{cm}^{-1}\right)$ : 3143, 3050, 2949 (C-H), 1650, 1520; ${ }^{1} \mathrm{H}$ NMR $\left(\mathrm{CD}_{3} \mathrm{COCD}_{3}\right): \delta 7.07(\mathrm{t}$, $1 \mathrm{H}), 7.34(\mathrm{t}, 1 \mathrm{H}), 7.38(\mathrm{t}, 1 \mathrm{H}), 7.45(\mathrm{t}, 1 \mathrm{H}), 7.65(\mathrm{~d}, 1 \mathrm{H}), 7.70$ $(\mathrm{d}, 1 \mathrm{H}), 7.75(\mathrm{~d}, 1 \mathrm{H}), 8.00(\mathrm{~d}, 1 \mathrm{H}), 12.101(\mathrm{~N}-\mathrm{H}) ;{ }^{13} \mathrm{C} \mathrm{NMR}$ $\left(\mathrm{CD}_{3} \mathrm{COCD}_{3}\right): \delta 119.95,121.49,123.43,126.73,127.56$, $128.05,130.07,131.50,132.09,133.56,137.03,150.78$, 159.84, 170.21; MS (m/z, \%): $[\mathrm{M}+\mathrm{H}]^{+} 426.9022$ (37.80), 428.9005 (78.44), 430.9016 (100), 432.9014 (51.88); Anal: C, 39.46; H, 2.02, N, 6.49.

\subsubsection{2-(4-Bromobenzoylamino)-1,3-benzotellurazole (2d)}

Colorless crystals, M.F.: $\mathrm{C}_{14} \mathrm{H}_{9} \mathrm{BrN}_{2} \mathrm{OTe}$; Yield: $67 \%$; M.W.: 428.73; M.P. (K): 509-511; FTIR $\left(\mathrm{cm}^{-1}\right)$ : 3133, 2952 (C-H), 1649, 1587, 1531 (aromatic), ${ }^{1} \mathrm{H}$ NMR $\left(\mathrm{CDCl}_{3}\right): \delta$ $7.17(\mathrm{t}, 1 \mathrm{H}), 7.39(\mathrm{t}, 1 \mathrm{H}), 7.52-7.56(\mathrm{~m}, 3 \mathrm{H}), 7.88(\mathrm{~d}, 1 \mathrm{H})$, $7.93(2 \mathrm{H}), 12.28(\mathrm{~N}-\mathrm{H}) ;{ }^{13} \mathrm{C}$ NMR $\left(\mathrm{CDCl}_{3}\right): \delta$ 119.07, $124.07,125.42,127.25,127.69,129.82,131.45,131.86$,
$133.14, \quad 145.58165 .75, \quad 171.75 ; \quad \mathrm{MS} \quad(\mathrm{m} / \mathrm{z}, \quad \%):[\mathrm{M}+\mathrm{H}]^{+}$ 426.9002 (37.80), 428.9005 (78.44), 430.9016 (100), 432.9014 (51.88); Anal: C, 39.47; H, 2.13 N, 6.63.

\subsubsection{2-(4-Methoxybenzoylamino)-1,3-benzotellurazole (2e)}

Colorless crystals, M.F.: $\mathrm{C}_{15} \mathrm{H}_{12} \mathrm{~N}_{2} \mathrm{O}_{2} \mathrm{Te}$; Yield: $64 \%$; M.W.: 379.87; M.P. (K): 423-424; FTIR ( $\left.\mathrm{cm}^{-1}\right)$ : 3133, 2952 (C-H), 1649, 1587, 1531 (aromatic), ${ }^{1} \mathrm{H}$ NMR $\left(\mathrm{CD}_{3} \mathrm{COCD}_{3}\right)$ : $\delta 3.59(\mathrm{~s}, 3 \mathrm{H}), 6.76(\mathrm{~d}, 2 \mathrm{H}), 6.81(\mathrm{t}, 1 \mathrm{H}), 7.10(\mathrm{t}, 1 \mathrm{H}), 7.45$ $(\mathrm{d}, 1 \mathrm{H}), 7.70(\mathrm{~d}, 1 \mathrm{H}), 7.98(\mathrm{~d}, 2 \mathrm{H}), 11.69(\mathrm{~N}-\mathrm{H}) ;{ }^{13} \mathrm{C} \mathrm{NMR}$ $\left(\mathrm{CD}_{3} \mathrm{COCD}_{3}\right)$ : $\delta$ 55.03, 113.71, 119.06, 123.23, 125.29, $126.78,126.94,130.51,130.63,131.59 ; \mathrm{MS}(\mathrm{m} / \mathrm{z}, \%)$ :

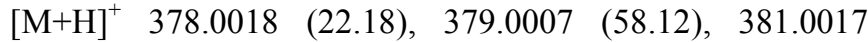
(92.60), 383.0034 (100); Anal: C, 47.35; H, 3.16; N, 7.31.

\subsubsection{2-Phenylamino-1,3-benzotellurazole (2f)}

Yellow crystals, M.F.: $\mathrm{C}_{13} \mathrm{H}_{10} \mathrm{~N}_{2}$ Te; Yield: 46\%; M.W.: 321.83; M.P. (K): 443-445; FTIR ( $\left.\mathrm{cm}^{-1}\right)$ : 2848 (C-H), 1593, 1437, 1207 (aromatic); ${ }^{1} \mathrm{H}$ NMR $\left(\mathrm{CD}_{3} \mathrm{COCD}_{3}\right): \delta 6.92(\mathrm{t}$, $1 \mathrm{H}), 7.06(\mathrm{t}, 1 \mathrm{H}), 7.30(\mathrm{t}, 1 \mathrm{H}), 7.36(\mathrm{t}, 2 \mathrm{H}), 7.63(\mathrm{~d}, 1 \mathrm{H})$, 7.76-7.79 (m, 3H), $9.44(\mathrm{~N}-\mathrm{H}) ;{ }^{13} \mathrm{C} \mathrm{NMR}\left(\mathrm{CD}_{3} \mathrm{COCD}_{3}\right): \delta$ $118.42,121.16,122.08,122.72126 .78,128.73,129.01$, $131.30,142.62,156.14,158.85 ; \mathrm{MS}(\mathrm{m} / \mathrm{z}, \%):[\mathrm{M}+\mathrm{H}]^{+}$ 319.9962 (22.63), 320.9952 (57.96), 322.9962 (92.82), 324.9979 (100); Anal: C, 48.68.13; H, 3.03; N, 8.64.

\subsubsection{2-(4-Chlorophenylamino-1,3-benzotellurazole (2g)}

Yellow crystals, M.F.: $\mathrm{C}_{13} \mathrm{H}_{9} \mathrm{ClN}_{2} \mathrm{Te}$; Yield: 44\%; M.W.: 356.28; M.P. (K): 463-465; FTIR (cm $\left.{ }^{-1}\right): 2841$ (C-H), 1592, 1547, 1438, 1202 (aromatic); ${ }^{1} \mathrm{H}$ NMR $\left(\mathrm{CD}_{3} \mathrm{COCD}_{3}\right): \delta 6.96$ $(\mathrm{t}, 1 \mathrm{H}), 7.31(\mathrm{t}, 1 \mathrm{H}), 7.36(\mathrm{~d}, 2 \mathrm{H}), 7.65(\mathrm{~d}, 2 \mathrm{H}), 7.78(\mathrm{~d}, 1 \mathrm{H})$, $7.84(\mathrm{~d}, 2 \mathrm{H}), 9.54(\mathrm{~N}-\mathrm{H}) ;{ }^{13} \mathrm{C} \mathrm{NMR}\left(\mathrm{CD}_{3} \mathrm{COCD}_{3}\right): \delta 114.05$, $118.40,120.08,121.35,122.31,126.84,129.01,131.37$, $131.79,141,77,155.51 ; \mathrm{MS}(\mathrm{m} / \mathrm{z}, \%):[\mathrm{M}+\mathrm{H}]^{+} 354.9560$ (18.20), 356.9565 (85.86), 358.9579 (100), 360.9563 (25.46); Anal: C, 44.11; H, 2.52; N, 7.98.

\section{Results and Discussion}

The choice of solvent for the synthesis of acyl isothiocyanates from the corresponding acyl chlorides and potassium thiocyanate appears to be critically important. At our hands, the use of acetonitrile as published [18] or toluene rather than benzene resulted in unsatisfactory yields. Purification of the prepared acyl isothiocyanates was greatly aided by their low polarities, which permitted the preparation of reasonably pure products by extraction with hexane, thereby keeping handling times and heat exposure to a minimum. While the preparation of thioureas derived from bis(2-aminophenyl) ditelluride proceeded smoothly, their ring closure was challenging. Initially, reductive ring closure using sodium borohydride was envisioned, with the intent to reduce the ditelluride moiety to the tellurole, followed by cyclization with elimination of hydrogen sulfide. A wide range of conditions was tested, including various solvents (dioxane, ethanol, acetonitrile, dimethylsulfoxide, hexamethylphosphoric triamide, acetic acid) and reducing agents (sodium borohydride, hypophosphorous acid, sodium 
hypophosphite, zinc dust, hydrazine hydrate). However, the resulting mixtures either underwent air oxidation back to ditellurides, indicating that the telluroles had failed to undergo cyclization, or extensive decomposition with precipitation of elemental tellurium. It should be noted that the mercury and Rongalite used for ring closure appear to be synergistic. The use of either reagent alone accomplished product formation in trace amounts only, as indicated by GCMS analysis. Satisfactory results, however, were only achieved by combining both. The mechanism of ring closure remains speculative, but the one proposed in Figure 3 is based on literature reports that mercury inserts into the Te-Te bond, generating species of type RTe-Hg-TeR [19-20]. It is probable that this initial insertion is followed by an intramolecular nucleophilic attack targeting the thiocarbonyl moiety and subsequent reductive desulfurization.

Several attempts to cyclize an analogous urea, prepared from bis(2-aminophenyl) ditelluride and phenyl isocyanate, were unsuccessful.

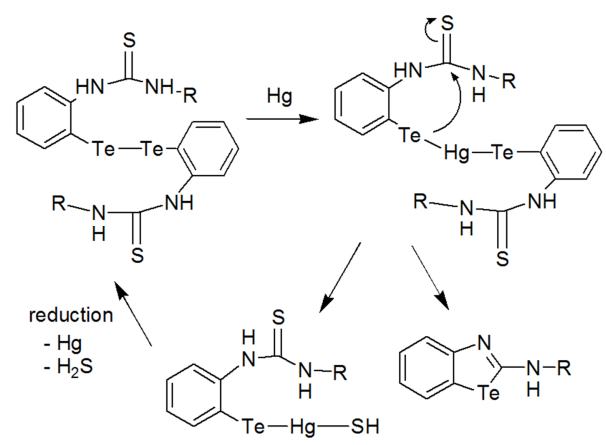

Figure 3. Plausible mechanism for the formation of 2-amino substituted 1,3benzotellurazoles in the presence of mercury.

An ORTEP plot obtained for 2-phenylamino-1,3benzotelluroazole reveals that this molecule forms dimers rather than the chain-like structures observed for various 2alkyl and 2-aryl substituted 1,3-benzotellurazoles [5] or ribbon structure found for 2-cyanomethyl-1,3-benzotellurazole [21]. Two molecules of 2-phenylamino-1,3-benzotelluroazole appear linked by intermolecular hydrogen bonds of $2.944 \AA$ and $2.982 \AA$, respectively, between adjacent endocyclic and exocyclic nitrogen atoms, as shown in Figure 4.

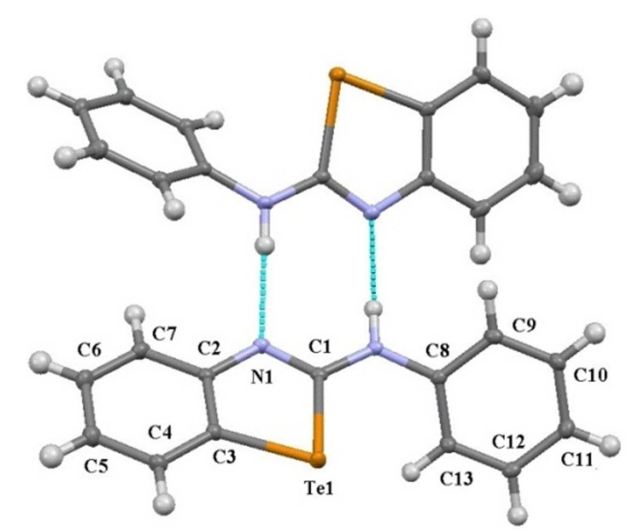

Figure 4. ORTEP plot of hydrogen bonded dimer of 2-phenylamino-1,3benzotellurazole $(2 f)$.
2-(Benzoylamino)-1,3-benzotellurazole showed similar crystal packing, again with hydrogen bonding between endocyclic and exocyclic nitrogens of adjacent molecules, as well as a $2.632 \AA$ intramolecular Te-O contact that renders the amide moiety coplanar with the heteroaromatic ring

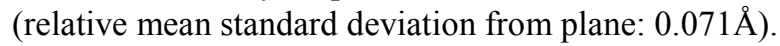

The X-ray crystallographic characterization of a third compound, 2-(4-chlorobenzoylamino)-1,3-benzo-tellurazole, revealed virtually identical behavior [22]. Similarly to other 1,3-benzotellurazoles, 2-acylaminobenzotellurazoles exhibit a high degree of air and light stability in both the solid and solution states. In contrast, 2-aminobenzotellurazoles tend to form orange colored decomposition products while in solution, making purification by recrystallization beyond $\sim 95 \%$ purity challenging.

No effort was made to isolate the intermediate thioureas prior to cyclization. They tend to degrade upon storage and should be prepared immediately before use.

Several attempts were made to prepare 2-alkylamino substituted 1,3-benzotellurazoles, starting with alkyl isothiocyanates and following the same procedure. While GC-MS analyses of the crude product mixtures indicated the presence of small quantities of the desired products, no useful yields could be obtained.

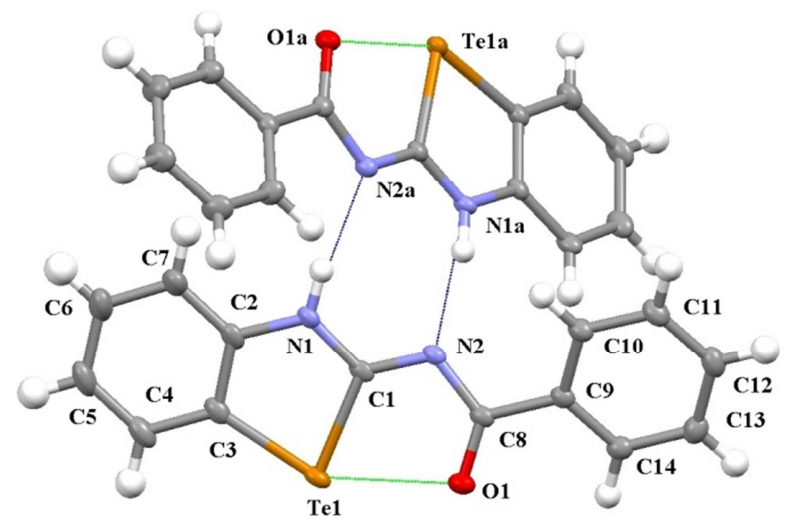

Figure 5. ORTEP plot showing hydrogen bonded dimers of 2-(4benzoylamino)-1,3-benzotellurazole (2a).

\section{Conclusions}

Previously unreported 2-acyl- and 2-arylamino-1,3benzotellurazoles were prepared to further investigate the tendency of substituted 1,3-benzotellurazoles to selfassemble to molecular wires. Their synthesis by treatment of bis(2-aminophenyl) ditelluride with aryl- and acyl isothiocyanates, followed by reductive desulfurization of the resulting thioureas represents a novel and convenient method of access to this class of compounds. However, it appears to be limited to the preparation of arylamino and acylamino derivatives and is unsuitable for the synthesis of alkylamino derivatives.

The self-assembly behavior of 1,3-benzotellurazoles is somewhat more varied than that of 1,2,5-telluradiazoles, which have long been known known to form molecular ribbons [23-24]. Those carrying isobutyl, phenyl, 2- 
phenylvinyl, 2-furanyl, 2-thienyl, 2-pyridyl, 3-pyridyl, 4pyridyl, and ferrocenyl substituents in position 2 were previously shown to self-assemble following a wire motif, in which each tellurium atom coordinates with a nitrogen atom on an adjacent tellurazole molecule [5]. Similar behavior was observed for 2-methylmercapto-1,3-benzotellurazole [25]. In contrast, the formation of strong hydrogen bonds between two adjacent molecules of the newly prepared 2-aryl- and acylamino-1,3-benzotellurazole appears to preclude their self-assembly to supramolecular structures, leading to dimers instead. This behavior is similar to that previously observed for 2H-1,4-benzotellurazin-3(4H)-one derivatives and 2,3dihydro-1,5-benzotellurazepin-4(5H)-one, which also were shown to form dimers by forming intermolecular hydrogen bonds rather than supramolecular assemblies [26].

While a paucity of structural data available for 1,3benzotellurazoles makes it difficult to generalize statements about their self-assembly behavior at this time, trends are emerging: the presence and nature of substituents in position 2 is critically important in determining the propensity of these compounds to form supramolecular assemblies. Compound carrying substituents in position 2 capable of strong hydrogen bonding form dimers while others form molecular wires.

\section{Acknowledgements}

We are grateful to the Department of Chemistry, University of Louisiana at Lafayette for material support of this work.

\section{References}

[1] A. F. Cozzolino, P. S. Whitfield and I. Vargas-Baca (2010). Supramolecular Chromotropism of the Crystalline Phases of 4,5,6,7-Tetrafluorobenzo-2,1,3-telluradiazole. Am. Chem. Soc. $132,17265-17270$.

[2] A. F. Cozzolino, Q. Yang, and I. Vargas-Baca (2010). Engineering Second-Order Nonlinear Optical Activity by Means of a Noncentrosymmetric Distortion of the $[\mathrm{Te}-\mathrm{N}]_{2}$ Supramolecular Synthon. Cryst. Growth Des. 10, 4959-4964.

[3] P. C. Ho, P. Szydlowski, J. Sinclair, P. J. W. Elder, J. Kübel, C. Gendy, L. M. Lee, H. A. Jenkins, J. F. Britten, D. R. Morim and I. Vargas-Baca (2016), Supramolecular Macrocycles Reversibly Assembled by Te...O Chalcogen Bonding. Nat. Commun. 7, 11299; doi: 10.1038/ncomms11299.

[4] G. Sanford, K. E. Walker, F. R. Fronczek and T. Junk (2017). Novel Organotellurium Heterocycles Derived From Bis (2Aminophenyl) Ditelluride. J. Heterocycl. Chem. 54, 575-579. doi: $10.1002 /$ jhet.2624.

[5] A. Kremer, A. Fermi, N. Biot, J. Wouters and D. Bonifazi (2016), Supramolecular Wiring of Benzo-1,3-chalcogenazoles Through Programmed Chalcogen Bonding Interactions. Chem. Eur. J. 22, 5665-5675.

[6] T. Junk, Chapter "Recent Advances in the Preparation and Characterization of Te, N-Containing Heterocycles", in "Tellurium: Properties, Uses and Research", Ed. D. Grey,
Nova Science Publishers, ISBN 978-1-53610-555-1, 2017 pp. 107-136.

[7] T. Junk, N. C. McMullen and F. R. Fronczek (2013). Organotellurium Chemistry: Remarkably Facile Preparation of Benzo-1,3-tellurazoles. J. Heterocycl. Chem. 50 (1), 120-124.

[8] S.-I. Fujiwara, Y. Asanuma, T. Shin-Ike and N. Kambe (2007). Copper(I)-Catalyzed Highly Efficient Synthesis of Benzoselenazoles and Benzotellurazoles. J. Org. Chem. 72 (21), 8087-8090.

[9] H. Ogura, S. Mineo and K. Nakagawa (1981). Studies on Heterocyclic Compounds XXXIV. Synthesis of 2-Substituted Aminobenzoxazoles with Nickel Peroxide. Chem. \& Pharm. Bull. 29 (6), 1518-1524.

[10] J. Sam and J. N. Plampin (1964). Benzoxazoles: Potent Skeletal Muscle Relaxants. J. Pharm. Sci. 53 (5), 538-544.

[11] M. D. Uher, J. Berkes, J. Lesko and L. Floch (1983). Reactions of Carbonyl Isothiocyanates with Nucleophilic Bifunctional Reagents. Coll. Czechoslovak Chem. Comm. 48 (6), 1651-1658.

[12] K. J. Irgolic, Houben-Weyl: Methods of Organic Chemistry, vol. E12b, Organotellurium Compounds, D. Klamann, Ed., Georg Thieme, Stuttgart, 1990, 152-153.

[13] A. Wroblewska and G. Mloston (2013). Synthesis of New Enantiopure Thioureas Derived from (S)-Proline. Phosphorus, Sulfur Silicon Relat. Elem. 188 (4), 509-511.

[14] A. S. Kuzovlev, E. V. Savinkina, V. V. Chernyshev, M. S. Grigoriev and A. N. Volov (2016). Copper and Palladium Complexes with Substituted Pyrimidine-2-thiones and 2Thiouracils: Syntheses, Spectral Characterization and X-ray Crystallographic Study. J. Coord. Chem. 69 (3), 508-552.

[15] F. Asghar, S. Rana, S. Fatima, A. Badshah, B. Lal and I. S. Butler (2018). Biologically Active Halo-Substituted Ferrocenyl Thioureas: Synthesis, Spectroscopic Characterization and DFT Calculations. New J. Chem. 42 (9), 7154-7165.

[16] L.-P. Duan J. Xue, L.-L. Xu and H.-B. Zhang (2010). Synthesis of 1-Acyl-3-(2'-aminophenyl) Thioureas as AntiIntestinal Nematode Prodrugs. Molecules 15, 6941-6947.

[17] A. Rana, N. Siddiqui, S. A. Khan, S. Ehtaishamul Haqueand and M. A. Bhat (2008). N-\{[(6-Substituted-1,3-benzothiazol2-yl)amino]carbonothioyl\}-2,4-substituted Benzamides: Synthesis and Pharmacological Evaluation. Eur. J. Med. Chem. 43 (5), 1114-1122.

[18] M.-J. Wang, X. Nan, G. Feng, Y. Gang, H.-T. Yu, G.-F. Hu and Y.-Q. Liu (2014). Design, Synthesis and Bioactivity Evaluation of Novel Acylthiourea Derivatives of Cantharidin. Ind. Crops Prod. 55, 11-18.

[19] E. S. Lang, R. A. Zan, C. C. Gatto, R. A. Burrow and E. M. Vazquez-Lopez (2002). Syntheses and Structures of New Tellurium-Mercury Clusters with Twelve- and Six-Membered Rings. Eur. J. Inorg. Chem. 2, 331-333.

[20] Y. Okamoto and Y. Takefumi (1971). The Reaction of Dioorganyl Diselenides and Ditellurides with Organic Mercury Compounds. A Convenient Method for the Synthesis of Unsymmetrical Organic Selenides and Tellurides. J. Organomet. Chem. 29 (1), 99-103. 
[21] G. Sanford, K. E. Walker, F. R. Fronczek and T. Junk (2016). CCDC 1423100: Experimental Crystal Structure Determination, DOI: $10.5517 /$ ccdc.csd.ccljrvgl.

[22] Mautner, F. A. and T. Junk, unpublished.

[23] V. Bertini, P. Dapporto, F. Lucchesini, A. Sega, A. DeMunno (1984). Actra Cryst. C 40, 653-655.

[24] A. F. Cozzolino and I. Vargas-Baca (2007). J. Organomet. Chem. 692 (13) 13, 2654-2657.
[25] G. Sanford, K. E. Walker, F. R. Fronczek and T. Junk (2017). Novel Organotellurium Heterocycles Derived from bis(2Aminophenyl) Ditelluride. J. Heterocycl. Chem. 54, 575-579. doi: $10.1002 /$ jhet.2624.

[26] J. P. Myers, F. R. Fronczek and T. Junk (2015). The First Crystal Structures of Six- and Seven-Membered Telluriumand Nitrogen-Containing (Te-N) Heterocycles: 2H-1,4-Benzotellurazin-3(4H)-one and 2,3-Dihydro-1,5-benzotellurazepin4(5H)-one. Acta Cryst. C72, 1-5. 